\title{
La représentation et la fonction de Montréal dans La canicule des pauvres de Jean-Simon DesRochers
}

\section{The Representation and the Function of Montreal in Jean-Simon DesRochers's La canicule des pauvres}

\author{
Veronika Černíková [53218@mail.muni.cz] \\ Jihočeská univerzita v Českých Budějovicích, République tchèque \\ Západočeská univerzita v Plzni, République tchèque
}

\begin{abstract}
RÉSUMÉ
Dans son premier roman, La canicule des pauvres, Jean-Simon DesRochers profite de son expérience de poète pour nous offrir un récit savamment construit autour de la vie dans une maison. La maison ressemble à la ville postmoderne où elle est située, à ce melting-pot de cultures et de natures humaines où la vie se déroule sans mode d’emploi. Sans être minutieusement décrite, Montréal imprègne le livre de sa présence chaleureuse et parfois même étouffante. Or, la réalité montréalaise que Jean-Simon DesRochers nous donne à lire ne lui sert que de prétexte à la création d’un roman assez complexe. En effet, la liste des pratiques postmodernes employées dans le roman paraît être complète comme si l'auteur voulait fabriquer une réflexion sur le roman postmoderne dont il se veut un exemple à suivre, lu par la masse, étudié par la critique et copié par la postériorité.
\end{abstract}

\section{MotS-CLÉS}

Montréal ; ville postmoderne ; roman postmoderne ; roman québécois ; métaroman ; décadence

\begin{abstract}
In his first novel La canicule des pauvres (2009) Jean-Simon DesRochers makes the most of his experience with poetry to offer us a postmodern narrative ingeniously constructed and focused on the life in one single house. This house is a little bit like Montreal itself; it resembles the postmodern city, this melting-pot of cultures and human natures where the life doesn't follow any schedule. Without any detailed description, the metropolis permeates the whole book with its hot and sometimes even stifling presence. However, the Montreal postmodern reality is but a pretext for the creation of a complex novel introducing all of the postmodern techniques as if the author wanted create a perfect example of the postmodern novel which would be read by the masses, analysed by the critics and copied by the epigones.
\end{abstract}

\section{KeYWORDS}

Montreal; postmodern city; postmodern novel; Quebec novel; metafiction; decadence

REÇU 2015-08-31 ; ACCEPTE 2016-06-30 
Nombreux sont les récits qui chantent les beautés de Montréal, cette incarnation même de la ville nordique. Or, l'image décadente offerte par Jean-Simon DesRochers dans son premier roman publié en 2009, La canicule des pauvres, est bien loin d'une telle représentation. ${ }^{1}$ La ville est captée pendant la période caniculaire. N'ayant pas de moyens pour s'offrir une climatisation, ce sont les pauvres qui en souffrent le plus ce qui explique l'attention particulière que l'auteur attribue aux descriptions de la pauvreté et des misères de la ville incandescente, de beaucoup préférées à l'illustration de ses beautés. En reconstruisant l'image de Montréal construite par Jean-Simon DesRochers dans La canicule des pauvres, nous proposons de réfléchir sur sa fonction dans le récit ainsi que sur les liens rattachant la ville au récit qui la représente. Mais avant la ville, regardons le récit lui-même.

\section{Un récit}

L'intrigue de La canicule des pauvres est construite autour de dix jours dans la vie de 26 occupants du "Galant », ancienne maison de passe transformée en immeuble locatif et située dans le Quartier latin. Le livre est divisé en dix parties dont chacune est limitée à l'espace d'une seule journée. Chaque partie est subdivisée en un nombre variable de chapitres qui suivent d'une manière chronologique les événements de la journée et qui sont introduits par les précisions de l'heure et de la température ressentie actuelles. Les chapitres, d'une brièveté étonnante, se succèdent dans un rythme infernal qui est encore accentué par la syntaxe : les phrases sont fréquemment courtes et simples ; la parataxe asyndétique domine les phrases complexes ; l'ensemble du texte est marqué par une forte présence d'ellipses, accumulations, énumérations et gradations. Par rapport à la structure du texte, l'auteur explique qu'il a voulu imiter l'effet du sac de chips : la lecture d'un chapitre donne envie au lecteur d'en lire un autre, un autre et un autre jusqu'à ce que le livre entier soit fini.

Avec la succession rapide de chapitres défilent rapidement les personnages : revendeur de drogues français, prostituée juive, tueuse à gages et sa victime, bédéiste japonais, membres séropositifs et anarcho-fouriéristes du groupe post-punk Claudette Abattage, pornographe et philosophe d'origine québéco-thailandaise, professeur d'université agoraphobe et homosexuel, aveugle, sportif, concierge floridien, immigrés latino-américains... bref, un melting-pot de ratés dont plusieurs mourants. Même si certains personnages sont favorisés, le livre n’a pour protagonistes que le climat et la maison. Cette dernière est organisée d'une manière parfaitement symétrique (voir l'image 1) : une croix composée de l'axe horizontal du couloir et de l'axe vertical de l'ascenseur et des escaliers divise tous les quatre étages en quatre secteurs identiques réunissant chacun deux appartements attenants. Lorganisation de la maison est alors basée sur la multiplication du chiffre deux qui porte en lui-même l'idée de la symétrie et de la polarité : 2 appartements attenants (l'axe du couloir et l'axe de l'ascenseur) ; 4 appartements de la moitié de l'étage (l'axe du couloir ou l'axe de l'ascenseur), 8 appartements de l'étage (la coupe horizontale), 16 appartements de la moitié de la maison (la coupe verticale), 36 appartements de la maison

1 Tous les renvois à la pagination se rapportent à l'édition Desrochers, J.-S. (2009). La canicule des pauvres. Montréal : Les herbes rouges. 
entière. La symétrie n’est rompue que par la présence du rez-de-chaussée avec le hall d’entrée, la réception et le restaurant.

Le Galant :

\begin{tabular}{|c|c|c|c|}
\hline 504 & $\begin{array}{c}502 \\
\text { Lulu, Bob, Mélina } \\
\text { Québec } \\
\text { groupe post-punk }\end{array}$ & 506 & $\begin{array}{c}508 \\
\text { Luc et Mike, Chloé } \\
\text { Québec, Brésil groupe } \\
\text { post-punk }\end{array}$ \\
\hline \multicolumn{2}{|l|}{2} & 505 & 507 \\
\hline $\begin{array}{c}404 \\
\text { Sarah } \\
\text { Canada } \\
\text { tueuse à gages }\end{array}$ & $\begin{array}{c}402 \\
\text { Takao } \\
\text { Japon } \\
\text { bédéiste }\end{array}$ & 406 & $\begin{array}{c}408 \\
\text { Jade } \\
\text { Juive } \\
\text { prostituée }\end{array}$ \\
\hline $\begin{array}{c}403 \\
\text { Monique et Christian } \\
\text { Québec } \\
\text { propriétaire/prostituée }\end{array}$ & $\begin{array}{c}401 \\
\text { Monique et Christian } \\
\text { Québec } \\
\text { propriétaire/prostituée }\end{array}$ & $\begin{array}{c}405 \\
\text { Trevor } \\
\text { Canada } \\
\text { mourant }\end{array}$ & $\begin{array}{c}407 \\
\text { Fanny } \\
\text { aveugle }\end{array}$ \\
\hline 304 & 302 & $\begin{array}{c}306 \\
\text { Tony } \\
\text { Québec } \\
\text { sportif }\end{array}$ & $\begin{array}{c}308 \\
\text { Kaviak } \\
\text { Québec/Thaïlande } \\
\text { pornographe/philosophe }\end{array}$ \\
\hline $\begin{array}{c}303 \\
\text { Marie-Laure } \\
\text { Québec } \\
\text { journaliste } \\
\end{array}$ & $\begin{array}{c}301 \\
\text { Daphnée } \\
\text { Québec } \\
\text { serveuse/actrice }\end{array}$ & 305 & $\begin{array}{c}307 \\
\text { Kaviak } \\
\text { Québec/Thaïlande } \\
\text { pornographe/philosophe }\end{array}$ \\
\hline $\begin{array}{c}204 \\
\text { Miguel et Juanita } \\
\text { Colombie } \\
\text { serveur et ouvrière }\end{array}$ & $\begin{array}{c}202 \\
\text { Guy } \\
\text { Québec } \\
\text { suicidaire }\end{array}$ & 206 & 208 \\
\hline $\begin{array}{c}203 \\
\text { Roméo et Henriette } \\
\text { Québec } \\
\text { cuisinier et mourante }\end{array}$ & $\begin{array}{l}201 \\
\text { Edward } \\
\text { Etats-Unis } \\
\text { concierge } \\
\end{array}$ & 205 & $\begin{array}{c}207 \\
\text { Claude } \\
\text { professeur d'Université }\end{array}$ \\
\hline & $\begin{array}{r}\text { ré } \\
\text { restauran }\end{array}$ & & \\
\hline & & & \\
\hline
\end{tabular}

Image 1

Bien que La canicule des pauvres soit son premier roman, Jean-Simon DesRochers n'est plus à l'époque un auteur inexpérimenté. En 2009, ses deux recueils de poèmes Lobéissance impure (2001) et Parle seul (2003) étaient salués par la critique et couronnés des prix littéraires dont le 
Prix Émile-Nelligan et deux autres romans dormaient dans son tiroir : Le Sablier des solitudes (2011) et Demain sera sans rêves (2013). En effet, la structure du roman, lorganisation symétrique de la maison, le travail systématique avec la syntaxe et les figures de style permettent de constater que l'auteur profite de son expérience préalable avec la poésie pour nous offrir un récit savamment construit et pourtant parfaitement accessible au plus large public.

\section{Une ville}

La quasi-totalité du roman se déroule à Montréal. Les personnages parcourent ses quartiers riches, avec des gratte-ciel, aussi bien que pauvres et habités par les immigrés, comme le petit Maghreb ou la petite Italie ; son centre avec le boulevard Saint-Laurent et la rue Saint-Catherine et sa périphérie dont notamment le quartier $\mathrm{O}$ de Brossard ; ses marchés Ontario et Jean Talon ; ses squares et carrés ; ses parcs. Plusieurs sont obligés de traverser la ville entière en prenant soit l'autoroute transcanadienne, soit le métro. La montagne et le fleuve avec les ponts ne manquent pas. C'est aussi le passé de la ville qui est évoqué : le boulevard Saint-Michel et les tramways des années 1940, le quartier Saint-Henri des années 1950, les Jeux olympiques des années 1970. Or, tous ces lieux, du présent et du passé, ne sont pas véritablement décrits, l'auteur se contente de mentionner leurs noms sans entrer dans les détails. C'est également le cas des vues panoramiques qui restent assez floues. Quand la ville est regardée du pont Champlain, le narrateur précise : «Sarah n’aperçut aucun gratte-ciel, encore moins la montagne. Le port restait une image diffuse, à peine visible. Le smog avalait tout, jusqu'à la fin du pont » (170). Une vue similaire s'offre à l'observateur du haut de la montagne : "Quant au panorama disponible, Tony resta sur sa faim. Un paysage gris et jaunâtre. Un smog épais. Quelques gratte-ciel flous en avant-plan et un horizon étroit, noyé dans la purée " (362). Si la ville se dévoile à l’observateur, ce n'est que dans les souvenirs de l'un des personnages : «Il se rappelle l’apparition de Montréal dans son pare-brise. Sa montagne, ses jeunes gratte-ciel, ses élégants ponts métalliques » (478). Cette image de la ville resplendissante appartient au passé lointain ; à présent, la ville est voilée par une épaisse couche du smog qui modifie la perception de la réalité.

Une attention particulière est néanmoins prêtée à la description du Quartier latin, notamment aux alentours du Galant : "Plutôt que de bifurquer à gauche dans sa rue pour aller vers l'artère commerciale, il va vers les vieux appartements transformés en condominiums pour jeunes bourgeois branchés. Il aime cette section, là où le pavé se rétrécit de manière à ne laisser passer qu'une voiture sous les grands érables » (31). Il s'agit d'un lieu merveilleusement bien situé, car «à côté des parcs, à côté du métro, à côté de la bibliothèque » (397). Le quartier est présenté comme un lieu plutôt agréable et familier.

Malgré son usure incontestable, Le Galant qui « sent le pauvre » (226) et qui est « trop jeune pour être vieux et trop usé pour être neuf » (762) témoigne d'une " intimité sordide » (762) qui en fait également un lieu plutôt agréable et familier. Selon Zach, le revendeur de drogues français, "l'âme d'un immeuble se cache toujours dans son escalier » (366). Observons alors avec Zach cette partie cruciale du Galant : «Zach use quelques minutes pour contempler la disposition moderniste de l'escalier du Galant. Il admire la vue en plongée où, cinq étages plus bas, il voit la couleur des tuiles couvrant le plancher du hall. Il regarde attentivement les marches 
de granit poussiéreux, sans aucune trace d'usure. Il caresse les montants en fer forgé torsadé de la rampe, analyse la résistance de la peinture noire qui les recouvre avec un ongle » (366). Son analyse lui permet de constater que « l'âme de l'endroit est celle d' 'une sympathique pouffiasse mal fardée et vieillissante, mais d’une poufiasse increvable...» (366-7).

Une ville, ce ne sont pas seulement les quartiers et les immeubles, ce sont aussi les gens qui les habitent. Bien évidemment, il y a la masse anonyme : une caissière nerveuse, un client, une fillette, un homme ventru, une étudiante aux haches larges, un étalagiste distrait, un gérant d'établissement et beaucoup de femmes jolies qui attirent l'attention des autres passants. Sinon, ce sont les marginaux qui peuplent les rues de Montréal : «Durant le jour, les sans-abri avaient laissé la place aux skinheads, hippies, skaters, rappeurs, alter-mondialistes, pacifistes et $\mathrm{BCGB}^{2}$ néolibéraux en tenue de week-end » (569). Ce sont aussi les marginaux des quatre coins du monde qui habitent Le Galant. Takao, le bédéiste japonais en quête des personnages pour sa nouvelle $\mathrm{BD}$, s'adonne à observer ses voisins du Galant : "J'en conviens, c'est plus de matériel que j’espérais récolter dans mes premiers jours. Ou bien le Canada est un pays de cinglés, ou bien je suis tombé dans une mine de diamants » (265). Il est d'ailleurs persuadé que les Canadiens sont « les porteurs génétiques d'une décadence» (264).

Plusieurs autres éléments complètent l'image de la ville : les objets, les plantes et les animaux, les sons et les odeurs. Les objets qui traînent dans les rues de Montréal sont de deux types principaux : les moyens de transports et les déchets. Le premier groupe est tout d'abord représenté par les voitures de marques et de catégories différentes : de la Audi TT à la Smart, du Dodge Caravan à la BMW décapotable. Qu’elles soient neuves ou rouillées, elles « rajoutent une épaisse voute de fumée bleue au smog verdâtre » (82). Non seulement elles polluent, elles sont dangereuses : «Bob ne voit pas la Audi TT qui grille un feu rouge en accélérant ferme. Chloé non plus. Bob pédale en la regardant. Elle le regarde avancer. Le type de la Audi compose un numéro sur son cellulaire. [...] Chloé voit la part aérienne de l'accident : le vélo de Bob propulsé sur une Smart garée en parallèle, Bob qui disparaît sous la Audi » (199). Et finalement, elles dérangent par leur bruit qui devient insupportable quand leur système d'alarme est activé en absence du propriétaire. Le second groupe est constitué des objets dont personne n'a plus besoin. Dès le premier chapitre, un percolateur qui ne marche plus est jeté par la porte-fenêtre du quatrième étage et heurte le toit d'une voiture brune. Dans une ruelle, une pile de matelas jaunis cache une télévision lourde et une table ; dans une autre, « il y a un tas de détritus dominé par un frigo, des chaises cassées, un matelas taché. Rebuts de déménagement, ça finira jamais, cette pollution... » (110) constate l'un des personnages. Un chapitre entier est consacré à la description du ramassage des ordures y compris l'énumération des déchets : des vélos rouillés, du verre cassé, les restes des repas, un matelas, un fauteuil de vieillard, un petit aquarium, des jouets, un barbecue, des plantes, une charogne affreuse, etc.

Parmi les plantes dont la présence est dans le roman remarquée dominent les arbres. La première mention est nettement positive, car le regard du narrateur est posé sur « les grands érables » (31). Hélas, dès la page suivante, le mauvais état de la verdure montréalaise est révélé, les arbres sont « ravagés par les maladies et de mauvais émondeurs » (32), ils sont « figés dans le

2 BCBG est une siglaison de «Bon chic, bon genre » qui désigne un style vestimentaire associant un raffinement extérieur à la culture et à l'éducation. 
smog» (523) où ils étouffent; même le gazon du parc est jauni. La situation des animaux n’est pas meilleure. Outre les pigeons bien nourris et un merle tranquille, soit les animaux meurent devant les yeux de leurs observateurs, tel l'écureuil qui se fait griller par un transformateur électrique ou le moineau qui se fait avaler par un chat ; soit ils sont en train de décomposer, tel le chien que quelqu’un a jeté à la poubelle et dont la tête, rongée par les larves de mouches, éclate sous le compacteur.

La trame sonore du roman mêle la musique à un éventail de bruits désagréables. Par exemple Idiotheque de Radiohead donne le rythme à l'action qui aboutit à l'accident : trois travailleurs suivent le rythme des cymbales avec leur marteau, les «femmes asiatiques se parlent en sautillant, presque en marquant le rythme " (198) et l'accident lui-même " prend la forme d'une succession de bruits. Chloé entend le crissement des pneus, le cri de Bob, un hurlement de douleur, la chanson de Radiohead, le klaxon de la Audi » (199). Les bruits de la rue, «klaxons, motos, gueulards, impatients, schizophrènes, enfants, ambulances, pompiers » (113) et les musiques, « celles des voitures sur la rue, des appartements voisins » (216) entrent sans cesse par les portes-fenêtres de l'immeuble. Le pire est le système d'alarme qui assomme les habitants du Galant pendant des heures entières : "Klaxons, sirènes, sifflements, grincements, couinements. Une collection de bruits aigus avoisinant les cent décibels passés en boucles de quatre secondes » (446). « De la tranquillité, du silence. C’est un luxe dans une grande ville » (444).

Pareillement aux sons, les odeurs de la ville offrent un mélange des arômes et des relents : «Il perçoit ensuite le fumet d'une cuisson de viande entremêlée à la puanteur d'une toiture dont on refait le goudron. Derrière cette masse olfactive, il perçoit un effluve singulier qui lui pose problème, celui d'une chair en perdition, une odeur d'animal mort... Il y a aussi l'odeur huileuse d'un restaurant qui se mélange avec les détails piquants de l'air. Un air acide, enfumé, lourd de pollens fleuris et d'odeurs d'essence brûlée. Il y a autre chose aussi... Un alcool volatil, résultat du travail de la grande distillerie de la ville. Une odeur de rhum industriel » (215-6). Les odeurs de la ville amènent l'un des personnages à la conclusion que « presque tout sent mauvais» (445).

L'image de la ville ne peut pas être reconstruite sans prendre en considération l'influence du climat. Par la bouche du personnage du créateur, le bédéiste japonais, l’auteur présente son idée qu'il y a un lien étroit entre le climat et les gens, que les gens en sont façonnés : "Ce climat ressemble à ses habitants... Instable, hypocrite... exagéré... souvent stagnan» (669). Le climat, c'est tout d'abord la canicule qui « joue la faucheuse dans la ville» (592) : « Il fait trop chaud depuis dix jours. Record de température par-dessus record de température. Les plus faibles meurent, les plus forts suent» (613). Mais la chaleur n'est pas le plus grand problème, c'est le smog qui l'accompagne, cette « soupe épaissie, grisâtre » (170) qui avale tout, même le ciel : "il n’y a pas de ciel. Que du smog. Gris. Jaune. Lourd. »(106) et qui déforme la perception de la réalité : "l'épaisseur de l'air déforme ce qui est visible, l'humidité colle à la peau, le smog laisse un goût dégueulasse dans la gorge, c'est presque complet... " (445). Après dix jours de la canicule, le smog est devenu « un état banal, une part du quotidien qui s'insère dans les conversations ordinaires parmi les mots argent, vitesse, sexe »(613). Le smog à Montréal incarne la pollution du monde : "Des reportages diffusés hier laissaient courir la rumeur d'un risque de pannes électriques dans les grandes villes américaines aux tours de verre, là où la climatisation est devenue vitale. 'Pour créer de l'énergie et fabriquer du froid, on brûle plus de gaz et de charbon. 
Malheureusement, en procédant de cette manière, on épaissit la couche de smog, on accélère le réchauffement climatique... c'est un cercle vicieux terrible... Il faudra bien s'en sortir un jour...’ (613).

Montréal de La canicule des pauvres est un melting-pot de nations, de cultures et de natures. Ses rues sont submergées des rebuts du déménagement et des voitures bruyantes et dangereuses. Produits d'une société de consommation, ils polluent la ville entière. La végétation urbaine en souffre autant que les gens. La présence des animaux et des personnes mourants ou en état de décomposition avancée accentue l'impression d’une ville décadente. En général désagréables, les sons et les odeurs font eux-aussi preuve de la pollution. La chaleur et le smog ajoutent à l'image de la ville quelque chose d'apocalyptique. Pourtant, cette ville, trop jeune pour être vieille, trop usée pour être neuve, porte en elle une beauté peut-être décadente mais aussi intime et familière puisque universelle. En effet, Montréal incarne le prototype d'une ville postmoderne : il est possible de la substituer à n’importe quelle autre métropole contemporaine. Si l'âme d'un immeuble se cache dans son escalier, l'âme de Montréal réside certainement dans Le Galant et la pollution de la ville peut être assimilée à celle du monde entier. C'est pourquoi l'un des personnages caractérise le panorama disponible du mont Royal comme une «vue incomparable sur la pollution du monde » (362). Et effectivement, La canicule des pauvres offre à son lecteur une vue identique.

\section{Un récit postmoderne pour une ville postmoderne}

Le fait que Jean-Simon DesRochers a choisi de représenter Montréal en tant que ville témoignant d'une décadence postmoderne nous amène à la supposition que le récit de La canicule des pauvres fasse également preuve de cette même décadence postmoderne. Dans son étude Le roman français postmoderne, Marc Gontard (2003) distingue trois grandes familles de tendances formelles de la littérature postmoderne : discontinuité, métatextualité, renarrativisation du récit. D’après Gontard (2003), la discontinuité « met au premier plan l'expérience de l'hétérogène, de l'altérité, du chaos, selon l'ontologie lyotardienne du Différend » (73-4). De cette manière, la discontinuité postmoderne s'oppose à " la pensée unitaire et totalisante de la modernité " (Gontard 2003 : 73). Les procédés qui appartiennent à cette famille relèvent du collage, de la fragmentation et de l'hybridation. La deuxième famille de tendances " apparait dans la mise en cause de la notion d'originalité qui relativise le pouvoir d'innovation " (Gontard 2003 : 74), tellement revendiqué par les modernistes. Le roman postmoderne est alors hypertextuel et pratique citation, réécriture, pastiche, métatextualité. Contrairement à la modernité, une distance ironique est nécessitée. La troisième famille de tendances correspond à "la recherche d'une nouvelle lisibilité » (Gontard 2003 : 74). Elle « se caractérise par une linéarité en trompe-l’œil, le recours carnavalesque à l'Histoire comme modèle de narrativité et la tentation de l'autofiction qui pervertit le repli narcissique du sujet par une mise en fiction de l'altérité du moi.» (Gontard 2003 : 74). Dans le texte qui suit, nous tenterons de vérifier à quel point La canicule des pauvres emploie les pratiques postmodernes, répertoriées par Marc Gontard.

Parmi les pratiques produisant « un effet massif de discontinuité » (Gontard $2003: 75)$ prime le collage qui « provoque des rapprochements inattendus en juxtaposant sur une même surface 
des éléments hétérogènes " (Gontard 2003 : 75). Le collage postmoderne implique " l'hétérogénéité radicale d'un monde rebelle à l'intention globalisante » (Gontard 2003 : 76) : les fragments hétéroclites sont « détournés de leur contexte d’origine et assemblés de manière aléatoire » (Gontard 2003 : 76). Ainsi fonctionnent de nombreux messages qui sont dans La canicule des pauvres juxtaposés sans aucun lien évident. Il s'agit notamment du zapping pratiqué par plusieurs personnages qui permet à l'auteur d'introduire dans son récit des bouts des émissions télédiffusées ou radiodiffusées :

Première chaîne : documentaire sur les gazelles ;

zap : vidéo de gangsta rap incluant voitures modifiées, vêtements Fubu, agressivité, filles de seize ans en bikinis ;

zap : télé-réalité, une femme complote contre une autre femme qui croit qu’un homme complote contre un autre homme ;

zap : télé-réalité, un laideron boutonneux paré d'un t-shirt Ohio State essaie de ne pas cracher ses cordes vocales en chantant I Will Always Love You;

zap : documentaire sur les hyènes qui emmerdent les lionnes lorsqu'elle chassent les gazelles dans la savane ;

zap : deux astronautes pissent sur Mars en rigolant ; Edward a déjà vu ce film...;

zap : baseball, les Braves servent une raclée aux Nationals ;

zap : documentaire sur les alligators des Everglades ;

zap : télé annonce, Dodge Polara 1963, pièce de collection, entièrement restaurée, on demande... (477).

D’après Gontard (2003), le zapping est « aujourd'hui la métaphore qui exprime le mieux la pratique postmoderne du collage »(77). Outre le collage audiovisuel et auditif, Jean-Simon DesRochers a recours au collage visuel en laissant ses personnages regarder les photographies, notamment celles qui ne leur appartiennent pas.

Proche du collage sont les inventaires, fort abondants dans La canicule des pauvres : de l'inventaire des prostitués et des passants jusqu'à l'inventaire des déchets et des odeurs en passant par l'inventaire des rues et des repas. À titre d'exemple, ne citons que l'inventaire des rues : « La collecte a lieu dans le quartier $\mathrm{O}$ de cette banlieue de la Rive-Sud. Par la vitre du camion, Tony regarde les noms des rues défiler : Océanie, Orsini, Odéon, Odessa, Oslo, Osaka, Oasis, Othello » (182) et celui des prostituées :

$19 \mathrm{~h}$ : Kim, minuscule asiatique spécialisée en prostate ;

$20 \mathrm{~h}$ : Tatiana, grande Russe blonde au pouvoir d'aspiration légendaire ;

$21 \mathrm{~h}$ : Justine, jeune étudiante aux seins refaits ;

$22 \mathrm{~h}$ : Stéfie, longues jambes, seins d'adolescente, bavarde, mais expérimentée ;

$23 \mathrm{~h}$ : Sabrina, dominatrice de courte taille, très recommandée, excellent service ;

Minuit : Alex, androgyne à la peau de lait et aux cheveux courts, amateur de tantrisme ;

$1 \mathrm{~h}$ : Ange-Marie, grande Haïtienne aux allures de top modèle décati ;

$2 \mathrm{~h}$ : Tatiana et Sabrina, la succion et le fouet ;

$3 \mathrm{~h}$ : Lynn, anglophone baptiste réactionnaire à tendance nymphomane ; 
$4 \mathrm{~h}$ : Justine et Lynn, le silicone et l'obsession ;

$5 \mathrm{~h}$ : Cassiopée, la meilleure pour faire voir les étoiles ;

$6 \mathrm{~h}$ : Pénélope, amateur du style grec, moyennant supplément. (149-150)

Le plus exhaustif est cependant l'inventaire des déchets qui occupe l'espace d'un chapitre entier et représente un véritable collage, un rassemblement aléatoire des vestiges de la société de consommation.

Une autre forme de discontinuité est l'écriture fragmentale où le fragment est « un produit de l'instant, c'est-à-dire du discontinu, soumis au hasard » (Gontard 2003 : 82). "C'est une écriture du chaos, du discontinu, qui introduit le vertige du particulaire et de l'aléatoire » (Gontard 2003 : 83). La canicule des pauvres fait penser à l'assemblage de fragments, un pêle-mêle de morceaux de vies. Contrairement à son modèle, La vie mode d'emploi, la composition de La canicule des pauvres ne suit aucune règle dépistable, elle paraît parfaitement aléatoire. Pour mettre en évidence ce contraste entre une œuvre de la modernité expérimentale et une œuvre postmoderne, la quatrième de couverture annonce : « la vie dans ce monde trop jeune pour être vieux et trop usé pour être neuf, c'est la vie, sans mode d'emploi. » Le caractère fragmental du récit va de pair avec l'effet du sac de chips mentionné ci-dessus. Bien que les événements soient racontés d'une manière chronologique, aucun lien logique ne les relie ni ne détermine leur suite : ils sont parfaitement interchangeables comme les chips d'un seul sac.

D'après Marc Gontard (2003 : 89), le collage et la fragmentation mettent en ouvre le chaos postmoderne. Le métissage, le troisième dispositif de discontinuité, fait entrer le principe d'hétérogénéité qui agit principalement au niveau du code, "puisque le récit relève de genres différents dans les cultures métissantes » (94), et au niveau des langues, "dans la mesure où les interférences linguistiques mettent en place une véritable hétéroglossie du texte » (94). Le métissage générique de La canicule des pauvres est multiple. Il s'agit tout d'abord d'un roman philosophique qui pose de nombreuses questions sur le fonctionnement du monde actuel. Les réflexions philosophiques s'insèrent dans le récit notamment par l'intermédiaire du personnage de Kaviak. Ses activités de pornographe lui permettent de consacrer une partie de sa journée aux méditations et à la philosophie : il tient simultanément un blogue pornographique www.kaviaksex.com et un philosophique www.kaviakmind.com. Aux abonnés de ce dernier, il offre des vidéos thématiques. Certaines touchent sa ville et son pays. Tel est le cas de la diagnose attribuée à Montréal, la schizophrénie fonctionnelle, qui entraîne une réflexion sur le sexe des peuples. D’après Kaviak et Takao, le bédéiste japonais, les francophones et les anglophones de Montréal forment un couple : «Anglais, il a conquis Français, il l'a dominé pendant deux siècles jusqu'à ce qu'il se révolte et demande l'égalité sous menace de divorce... Selon cette explication, le peuple canadien-français se comporte comme une femme moderne "(544). Certaines d'autres vidéos sont plus universelles. Ainsi, Kaviak révèle l'évolution du phénomène du changement qui est devenu positif dès qu'il n’est plus risqué. Or, « plus les changements sont possibles, plus nos contentements sont brefs... L'instabilité s'est transformée en constance, en loi élémentaire... et l’obéissance à cette loi a transformé la nouveauté en absolu moral...» (146). La nouveauté occupe l'esprit, entraîne les «vacances de la pensée » : « le changement, c'est une médication contre la réflexion...» (147). De plus, Kaviak quête l'origine philosophique du réchauffement climatique qu'il découvre dans la tradition chrétienne de distinction entre l'âme et 
le corps : « Selon le concept, le corps serait un véhicule temporaire pour l'âme : la 'vraie vie', elle, serait ailleurs. L'esprit serait pur et céleste, le corps serait vulgaire, terrestre. Dans ces conditions, pouvez-vous me dire quel impératif moral peut retenir les propriétaires d'âme de saccager leurs vulgaires territoires terrestres? Personnellement, je n’en vois aucune » (230). Le réchauffement climatique est d'ailleurs considéré comme preuve irréfutable de la décadence postmoderne, de «notre grandeur devenue déclin " (419). L'humanité évolue vers une nouvelle forme mais, d'après Kaviak, « la nouveauté, c'est rarement bon » (421).

Outre un roman sans faute philosophique, La canicule des pauvres est un roman pornographique. Le Galant est quand même une ancienne maison de passe et sa propriétaire est une ancienne prostituée. Mais ce n’est pas seulement le passé de l’immeuble qui soit sexualisé, c’est aussi son présent. Certains de ses habitants font partie de l'industrie du sexe : le pornographe Kaviak, son ancienne actrice Daphnée, la prostituée Jade, son client Trevor. La plupart tout simplement aime faire l'amour. La variabilité est presque étonnante : nous assistons à des scènes d'extrême tendresse mais aussi de violence augmentée, à des scènes de masturbation solitaire ainsi que de sexe de groupe. Les pratiques sexuelles sont parfois ordinaires, parfois empruntées à marquis de Sade et ses Cent vingt journées de Sodome dont s'inspire l'un des personnages. Les amants sont hétérosexuels aussi bien qu'homosexuels ou bisexuels. Non seulement La canicule des pauvres est imprégnée du sexe, son auteur y met en scène des réflexions théoriques sur le genre pornographique : «- Quand tu regardes un film porno, généralement, t'es seul. Tu regardes deux, trois, quatre, $\mathrm{X}$ personnes jouer avec leur corps. Le procédé gagnant à tout coup, c'est de présenter les acteurs habillés, les mettre en action et les dévêtir. [...] Regarder un film, XXX ou pas, c'est comme lire un livre, ça comble la solitude, ça efface les angoisses... c'est du rêve instantané...» (488). Jean-Simon DesRochers n’hésite pas à comparer les films porno aux livres comme s'il voulait montrer que les limites entre les genres, entre le haut et le bas n'existent plus. Ce qui compte, c'est la possibilité de s'identifier au personnage, car « le spectateur prend virtuellement la place de l’acteur » (488), et la sincérité : personne n’aime «baiser une femme qui fait semblant de jouir » (489). "Un film, c'est toujours faux... pour être crédible, il faut montrer quelque chose de plus intime que la réalité... Les vrais voyeurs cherchent pas un spectacle, ils cherchent des apparences de vérité...» (489). Avec l'idéal du film porno, l'auteur formule l'idéal qu'il essaie d'accomplir avec son roman; il définit les règles du genre qu'il explore.

L'histoire de maints personnages fait penser à un roman sentimental. Ne citons qu'un exemple pour tous. En 1969, Edward a quitté sa famille et sa Floride natale en quête de Gina avec qui il venait de vivre plusieurs jours de bonheur. Il l’a trouvée à Montréal, ils ont répété leur romance mais après un mois, Gina est disparue de sa vie à jamais : elle a quitté Montréal avec son mari sans prévenir Edward, sans laisser sa nouvelle adresse. Depuis ce moment-là, Edward ne cesse plus de penser à cette femme fatale, à la chercher dans les rues de Montréal. Trente-sept ans plus tard, il a l'impression de la reconnaitre dans la rue mais elle ne le reconnait pas. Il la suit, l'approche, comprend que ce n'est pas sa Gina, l'invite à dîner, lui raconte son histoire. Ils passent une soirée agréable et décident de se revoir en vue d'un nouvel amour.

La mise en scène d'une tueuse à gages qui s'installe, comme sa victime, au Galant rend possible l'introduction des scènes empruntées aux thrillers des plus dramatiques. La présence de plusieurs personnages séropositifs, des mourants, des suicidés et des autres perdants de l'immeuble, entraîne des scènes types des romans psychologiques. Le choix des personnages et du 
milieu va de pair avec le discours sociologique. Le rôle principal attribué au climat invite au discours écologiste. Bref, une grande diversité de personnages permet à Jean-Simon DesRochers de faire défiler, dans sa Canicule des pauvres, toute une série de genres et de discours, de créer un roman hybride, pluriel.

Le métissage linguistique de La canicule des pauvres ne cède en rien au générique. Il est vrai que la plupart des personnages parle le québécois ou éventuellement le joual. Néanmoins, les immigrés et leurs descendants emploient des mots, des expressions, même des discours entiers en d'autres langues. Tel est le cas de Zach, revendeur de drogue toulousain, qui parle le français métropolitain. Edward, concierge floridien, parle et pense en anglais. L'anglais est d'ailleurs fréquemment utilisé par d'autres personnages qui mêlent des expressions anglaises au discours en français québécois. Miguel et Juanita, immigrés colombiens, tout d'abord parlent entre eux en espagnol dont la traduction fait partie des notes en bas de page ; ensuite, les chapitres consacrés à Juanita sont présentés comme une "version traduite ". Tout en venant d'arriver à Montréal, le bédéiste japonais Takao parle et pense dans un français excellent. De point de vue langagier, son origine ne se manifeste que lors de l'appel à son éditeur au Japon et dans de rares moments où il incorpore dans son discours des expressions japonaises. Et finalement, étant d’origine juive, la prostituée Jade prononce une prière dans la langue yiddish. De plus, elle maitrise l'italien quelle utilise en parlant avec son ancien chef, l'Italien Angelo. Malgré un haut degré de l'hybridation linguistique due à la diversité des origines des personnages, le discours central du roman, à savoir celui du narrateur, unifie l'ensemble du texte. Même les chapitres consacrés aux personnages qui parlent rarement français, tel Edward dont la plupart des répliques et des pensées est en anglais, sont racontés en français. Cela permet à La canicule des pauvres d'être un texte langagièrement très diversifié et, à la fois, cohérent. Or, l'hybridation ne se limite pas chez Jean-Simon DesRochers au domaine générique et linguistique, elle touche également le domaine thématique car l'immeuble et avec lui la ville entière sont présentés comme un melting-pot de cultures et de natures humaines.

La deuxième famille de tendances du roman postmoderne, la métatextualité, est définie par Bernard Magné (1998) comme « l’ensemble des dispositifs par lesquels un texte donné désigne, soit par son dénotation, soit par connotation, les mécanismes qui le produisent » (33). Selon cette définition, il est possible de distinguer différents procédés métatextuels : le commentaire, l'autoreprésentation et la spécularité d'une part ; les pratiques de seconde main, à savoir l'intertextualité et l'hypertextualité, d'autre part. Dans La canicule des pauvres, les commentaires sont assurés par un narrateur omniscient. Il commente et dévoile ce que les personnages ne peuvent pas savoir : «Une blatte file sur le tapis à vive allure. Trevor bondit de sa chaise, traque l'insecte, l'écrase d'un vif coup de talon. Va chier... En retournant vers son Vaio, Trevor ne remarque pas l'absence de cadavre d'insecte sur le plancher ni sous sa chaussure DKNY»(103-4). Trevor ne peut pas le remarquer, puisque l'insecte est son hallucination. Le narrateur aime anticiper en révélant ce qui arrivera plus tard ou par contre n'arrivera point : « Takao devra s'y faire. Suki ne viendra pas l'envahir. Elle ne l'aurait pas plus visité s'il était resté dans son loft du Minami, mais ça, Takao ne le croit pas » (101). Le narrateur est assez narcissique, il aime montrer son intelligence qui dépasse largement les limites de ses personnages : « Le cri de Marsouin provient d'un réflexe neural qui échappe à ce qui lui reste de conscience. Un neurologue estimerait que ce patient est cliniquement mort, que le cerveau ne fonctionne plus, que ce cri est léquivalent 
d'un spasme post mortem. Le Marsouin contredirait cette science s'il en avait la capacité » (138-9). Le narrateur est impassible, ironique et parfois même cynique. Il décrit le suicide du Marsouin et la décomposition de son corps avec une précision chirurgicale et sans manifestation de moindre émotion. Le Marsouin une fois mort, le narrateur n’hésite pas à remarquer la présence des mouches : «Cinq mouches domestiques se régalent d'une tache de mélasse sur un comptoir, deux se nourrissent autour de la cuvette des toilettes, trois autres économisent leur énergie après une lutte contre la perfide technologie des fenêtres. Ces mouches s'activeront bientôt. D’ici quelques minutes, elles découvriront un territoire inespéré pour engendrer une nouvelle génération vorace » (139). Contrairement au Marsouin, le sort des mouches occupe le narrateur : «Elles arpenteront le corps du Marsouin avec la satisfaction biologique du devoir accompli. Malgré tout, les mouches demeureront sur le qui-vive, effrayées par la possibilité d'être repérées par un prédateur. Elles ne trouveront aucun repos dans l'abondance » (154-5). La canicule des pauvres reprend le narrateur de type balzacien sauf quelle en fait un être narcissique et cynique, touché davantage par le destin d'une mouche que d'un être humain.

Un autre procédé par lequel le texte met en évidence sa littérarité tout en dévoilant son propre fonctionnement est l'autoreprésentation, définie par Janet M. Paterson (1982) comme un " processus selon lequel un texte se représente » (177). Son élément de base est la représentation de l'auteur ou, au moins, la mise en scène de la figure du créateur. Dans La canicule des pauvres, l'auteur ne s'exhibe pas vraiment ; il préfère inventer les personnages des créateurs. Il y a bien évidemment Kaviak dont les blogues sont suivis régulièrement par des centaines d'abonnés. Les deux blogues sont autobiographiques puisqu'il y présente ses propres prouesses, sexuelles et spirituelles, seulement son blogue pornographique est de beaucoup plus suivi que le philosophique. Néanmoins, Kaviak ne représente pas la principale figure du créateur ; c'est Takao, le bédéiste japonais dont la gloire dépasse les frontières du Japon. Kaviak est d'ailleurs l'un de ses admirateurs et il est fort surpris de le trouver à sa porte. Il y a deux raisons qui amènent Takao à Montréal. D’une part, il veut fuir son ancienne petite-amie qu'il a laissée à Osaka après l'avoir abandonnée dans le dernier volume de sa BD : «Suki allait apprendre la fin de leur couple en lisant son gekiga, qu'il la laissait, car elle nétait plus intéressante, tant pour lui que pour son histoire. Trop banale, avait-il écrit » (101). Tout comme Kaviak, Takao est le partisan de l'autobiographie. D'autre part, il cherche de l'inspiration qu'il ne trouve qu'à la fin du roman; c'est l'orage qui la lui apporte : «Mon personnage principal, ce sera le climat. Pas moi...le climat. C'est bien mieux. Il sera seul à être de toutes les scènes...il sera la goutte de sueur sur un front, le thermomètre accroché à une porte, un groupe de personnages dévêtus, un ventilateur qui ne remue plus rien... [...] Dans les mois qui suivront, sans suivre d'horaire ni de programme particulier, Takao visitera chaque occupant du Galant »(670-1). Ce nouveau projet de Takao correspond parfaitement à celui de La canicule des pauvres : le rôle principal est attribué au climat et le lecteur visite successivement, sans un programme particulier, tous les occupants du Galant pour découvrir les histoires de leurs vies.

L'analogie entre le projet scriptural du personnage et le roman qui le contient fait preuve d'une autre manifestation de la métatextualité : la mise en abyme. Définie par Lucien Dällenbach (1977) comme « toute enclave entretenant une relation de similitude avec l'œuvre qui la contient » (18), cette pratique spéculaire permet au texte de dévoiler encore une fois sa propre pratique significative. Le projet de Takao n'est pas la seule mise en abyme de La canicule des pau- 
vres. Henriette qui est en train de mourir d'un cancer du poumon est dépendante de sa télévision, car elle lui permet d'échapper à la réalité. Chaque jour, elle regarde un téléroman où un docteur tombe amoureux d'une femme dont l'identité reste incertaine. Henriette meurt avant de savoir si cette femme est la fille du docteur comme elle s'en doute. Une histoire analogue se déroule dans le roman. Un jour, Kaviak accueille chez lui une jeune femme qui le fascine. Elle arrive pour réaliser un film porno avec lui mais découvre qu'elle n'en est pas capable et se sauve. D'une certaine manière, Kaviak tombe amoureux d'elle. En même temps il se pose la question si ses parents biologiques qu'il n'avait jamais connus ne pouvaient pas avoir aussi une fille. Son visage qui ressemble étrangement au sien le hantera dans ses songes et il rêvera de la revoir. Il est à noter que les deux analogies relient le roman à deux genres mineurs et populaires : la BD et le téléroman. Encore une fois, les limites entre les différents genres, entre le haut et le bas se voient effacées.

Le commentaire, la figure du créateur et la mise en abyme sont des procédés métatextuels dénotatifs ; par leur intermédiaire le texte ne renvoie qu’à lui-même. Il en exhibe sa littérarité, son autoconscience. À l'autre échelle de la métatextualité se trouvent les stratégies connotatives par lesquels le texte renvoie à d'autres textes : l'intertextualité et l'hypertextualité. Tout en jouant avec l'effet de seconde main, ils mettent en cause la notion d'originalité. Parmi les pratiques intertextuelles employées dans La canicule des pauvres priment les citations et les allusions. Le plus souvent, les personnages écoutent leurs chansons préférées ou ils les repassent dans leurs têtes. Ils écoutent les genres les plus variés, de la musique classique au post-punk en passant par le concours de Star Académie. La musique est d'ailleurs partout, elle arrive de la rue, des appartements voisins, de la radio. La musique est tellement importante qu'elle rythme la perception de la réalité, tel est le cas de Idiotheque des Radiohead qui sert de fond sonore à l'accident de voiture. Si la musique est plus ou moins omniprésente, les autres arts le sont de beaucoup moins. Maints habitants du Gallant regardent régulièrement la télévision mais on ne mentionne que plusieurs films : Dogville de Lars von Trier, Last night de Don McKellar, Todo sobre mi madre de Pedro Almodóvar et Limelight de Charlie Chaplin. Ce sont davantage les acteurs, ou plutôt les actrices, qui sont retenus. En effet, Daphnée, la copine du revendeur de drogue, est souvent comparée à Nicole Kidman. En se prenant pour actrice, elle aime imiter le comportement des actrices célèbres, telles Kate Moss, Meryl Streep, Winona Ryder et beaucoup d'autres. Encore moins les personnages lisent. Une exception représente assez paradoxalement Fanny, femme aveugle, qui lit les essais populaires de Stephen Hawking et d'Anne Cauquelin. Jade, la prostituée, devient lectrice contre sa volonté. Son client Trevor dont le cerveau est abîmé par le cancer la paie pour lire Les cent vingt journées de Sodome. Un homme noir, entrevu dans le métro, lit Hegel. Takao, le bédéiste, est comparé à Beckett puisqu'il a également décidé de fuir son pays natal et a choisi de créer en français.

Dans la plupart des cas, les personnages font allusion à la culture de masse : les chansons populaires, les acteurs célèbres, les émissions télédiffusées et radiodiffusées. Or, l'intertextualité dans La canicule des pauvres est travaillée à deux niveaux différents. Outre les renvois à la culture de masse vécue par les personnages, Jean-Simon DesRochers incorpore dans son texte des jeux intertextuels plus sophistiqués et parfois ironiques. Par les titres des chapitres, l'auteur invite le lecteur à jouer avec lui tout en travaillant avec son horizon d'attente. Ainsi le chapitre intitulé LA PHILOSOPHIE DES LUMIERES ne parle pas de la philosophie du $18^{\mathrm{e}}$ siècle, Kaviak y 
philosophe sur la lumière et son pouvoir d’accélérer la décomposition. Certains titres reprennent les titres des ouvres classiques de la culture mondiale, comme LA DOUCE VIE (La dolce vita de Fellini), LA PLUIE ET LE BEAU TEMPS (le recueil de poèmes de Prévert), IDIOTHEQUE (la chanson de Radiohead), LE DEJEUNER SUR L'HERBE (le tableau de Manet). Certains d'autres les reprennent tout en les modifiant d'une manière ironique, tels LA DECOUVERTE DU TEMPS PERDU (À la recherche $d u$ temps perdu de Proust), LE VIEIL HOMME ET LE RIEN (The Old Man And The Sea de Hemingway), ELOGE DE LA RAISON CYNIQUE (Eloge de la raison de Voltaire), CETTE FEMME EST UNE AUTRE (l'affirmation rimbaldienne « je est un autre »), MONIQUE ON THE ROAD (On The Road de Kerouac). Par ses jeux intertextuels, l'auteur fait des clins d'œil au lecteur averti, il veut lui montrer à quel point il est cultivé et qu'il dépasse largement le niveau de ses personnages, car ses allusions ne concernent pas la culture populaire mais renvoient aux œuvres classiques qui ont marqué leur temps. Son narcissisme est d'autant plus frappant que le titre du chapitre PARLER SEUL fait référence à son deuxième recueil de poèmes, Parle seul (2003). Il paraît que l'auteur place son œuvre et lui-même à côté de ces géants.

La dernière pratique de la famille métatextuelle, l'hypertextualité, est définie par Gérard Genette (1982) comme " toute relation unissant un texte B (hypertexte) à un texte antérieur A (hypotexte) sur lequel il se greffe d'une manière qui n'est pas celle du commentaire» (11-12). Jean-Simon DesRochers procède de cette manière sauf qu'il ne greffe pas son roman sur un seul hypotexte mais sur plusieurs. Premièrement, il y a Le Décaméron de Boccace. Tout comme ce roman, La canicule des pauvres se déroule en dix jours, elle regroupe un ensemble considérable d'histoires dont le ton est souvent érotique et qui fonctionnent d'une manière indépendante - le roman peut être lu en diagonale en suivant l'histoire de tel ou tel personnage. Il y a néanmoins une grande différence entre les deux romans : la structure strictement symétrique du roman de la Renaissance a été remplacée par le chaos postmoderne. Chaque jour réunit un nombre variable de chapitres qui sont, paraît-il, organisés d'une manière aléatoire et l'histoire de chaque personnage s'étale sur l'espace non seulement de plusieurs chapitres mais aussi de plusieurs jours.

Deuxièmement, La canicule des pauvres se greffe sur Le père Goriot de Balzac. En effet, Le Galant ressemble en beaucoup de points à la pension Vauquer. Les deux immeubles locatifs sont situés au centre de la ville et pourtant ils sont assez miteux et délabrés. Leur propriétaire est une cinquantenaire suspecte. Leur rez-de-chaussée abrite soit une salle à manger, soit un restaurant, les deux meublés d'une manière vieillotte. Plusieurs étages sont occupés par des locataires plutôt pauvres. Les chambres dans les étages supérieurs sont encore plus misérables que celles des étages inférieurs car plus petites ou plus chaudes. De plus, les romans suivent les histoires personnelles des habitants de la maison qui n'aboutissent pas toujours à une fin heureuse. Un point commun représente aussi le narrateur qui est dans les deux cas omniscient et qui ne se prive pas de commenter le comportement des personnages, d'exprimer son opinion ou de montrer son point de vue.

Troisièmement, Jean-Simon DesRochers construit son roman à partir de La vie mode d'emploi de Georges Perec. D’une part, il y a l'immeuble qui se trouve au centre d'intérêt des deux romans. Les pièces chez Perec et les appartements chez DesRochers sont organisés de telle façon qu'ils ressemblent à une sorte d'échiquier, seulement celui de La canicule des pauvres ne dispose 
que de la moitié des cases. La narration se déplace d'une pièce à l'autre et il est toujours facile de deviner dans quelle pièce ou appartement se déroule l'action. Néanmoins chez DesRochers, les indications des numéros des appartements sont parfois trompeuses. Si à la page 117 Le Marsouin habite au numéro 202, à la page 139 il est hébergé au numéro 201 sans avoir déménagé. Difficile de savoir si Jean-Simon DesRochers s'inspire de Perec et de son exception à la règle, de Robbe-Grillet qui dans ses romans insère des éléments rendant impossible la reconstruction temporelle et spatiale du récit ou s'il s'agit tout simplement d'une inattention. En outre, la narration de La vie mode d'emploi est organisée d'une manière systématique, inspirée par la règle du jeu d'échecs : pour se déplacer d'une pièce-case à l'autre, Georges Perec suit le mouvement du cavalier. Comme nous l'avons déjà mentionné, la narration de La canicule des pauvres est par contre aléatoire. Pourtant, il paraît que Jean-Simon DesRochers s'est laissé également inspirer par la règle d'un jeu mais ce jeu, il l’a inventé lui-même tout en précisant sa règle : « N’oublions pas la méthode... dresser l'inventaire des sujets disponibles : sept hommes et cinq femmes. Pas plus qu'une minute et quart par sujet... et une maxime inventée à la fin... sinon, c'est pas une partie de Stills, c'est rire des gens... » (335). En effet, les premiers chapitres ressemblent à l'inventaire des habitants du Galant, ensuite l'auteur relate leurs histoires personnelles tout en ne dépassant pas l'espace de plusieurs pages. Les maximes sont remplacées par un arrière-plan philosophique et par un dénouement tantôt tragique, tantôt heureux. Ainsi l'auteur dévoile non seulement le passé des personnages mais aussi leur avenir. Même si le ton est parfois ironique, nous n'avons jamais l'impression que l'auteur rit de ses personnages. Semblablement à une partie de Stills, le résultat de cette pratique scripturale est une série de portraits vivants.

Sans aucun doute, la relation principale entre l'hypertexte et ses hypotextes consiste dans le fait que tous les personnages se réunissent par hasard et d'une manière plus ou moins temporaire dans un seul immeuble, et que leur réunion n'est qu'un prétexte à raconter les histoires. Or, le choix des hypotextes a encore une autre motivation : ils sont des représentants les plus marquants des grands tournants dans l'évolution du genre romanesque. Le Décaméron, à la limite du recueil de nouvelles et du roman, est l'une des œuvres fondatrices de la prose mondiale. Le père Goriot est une incarnation parfaite de l'âge d'or du roman, un prototype du roman traditionnel. Avec La vie mode demploi, le roman entre dans la période moderniste qui aboutit à la crise du genre. La fin des avant-gardes déclare même sa mort due à son illisibilité. En se plaçant dans la même lignée, La canicule des pauvres se donne pour un exemple du roman postmoderne qui apporte la renaissance du genre redevenu lisible et attirant sans être banal. En tant que roman postmoderne, La canicule des pauvres ne refuse ni la tradition, ni la modernité sans les imiter aveuglément. Elle regarde en arrière, elle cherche son inspiration dans le passé tout en noubliant jamais de dépasser ses modèles. Son dépassement consiste notamment dans le remplacement de la symétrie et du système par le chaos postmoderne et dans la recherche d'une nouvelle lisibilité.

Le retour à la lisibilité est le résultat de la renarrativisation du récit constituant la troisième famille de tendances postmodernes. Cette renarrativisation consiste dans la revisitation des formes du passé « d'une façon non innocente " pour emprunter l'expression d'Umberto Eco (1985 : 77). Le retour à la narration se déroule de trois façons possibles. La première est marquée par la volonté de raconter une histoire. Parmi les formes du passé revisitées figurent d'une part celles qui étaient longtemps prises pour les plus nobles : le roman historique, mythopoiétique, 
psychologique, philosophique, social... ; d'autre part celles qui appartenaient jadis aux genres mineurs, populaires, indignes d'attention des grands artistes : le roman policier, d'aventures et d'espionnage, sentimental et érotique... Contrairement aux autres auteurs postmodernes, tel Jean Echenoz ou Tanguy Viel, Jean-Simon DesRochers ne s'adonne pas à réécrire, à revisiter un seul genre. Son dépassement postmoderne consiste dans son aspiration à offrir au lecteur un amalgame où s'allie, sans préférence ni axiologie, le haut avec le bas, le roman philosophique et social avec le thriller sentimental et érotique.

La deuxième modalité de la renarrativisation est le retour à la réalité, exclue de la littérature par les dernières avant-gardes. Il ne s'agit pas néanmoins du retour au réalisme. La littérature postmoderne ne veut plus reproduire le réel, elle tente de souligner son non-sens. Une attention particulière est prêtée à la mise en œuvre de son dysfonctionnement qui entraîne fréquemment l'imaginaire de la fin. Le monde tel qu'il est présenté dans La canicule des pauvres est en pleine crise, il est imprégné de la violence, de la décomposition, de la maladie et de la mort. Les gens qui l'habitent sont les marginaux : les échoués, les drogués, les prostituées, les homosexuels, les immigrés, les handicapés, les malades et les mourants, les vieillards, les chômeurs et les artistes. Souffrant du réchauffement climatique, le monde fait preuve d'une usure et d'une décomposition avancées. Les réflexions philosophiques sur les phénomènes du changement, de la décadence et du déclin mettent à nu les mécanismes du (dys)fonctionnement du monde dont la fin paraît inévitable.

La troisième voie de la renarrativisation consiste dans le retour au sujet qui est le résultat du narcissisme de la société postmoderne. Il n’est pas étonnant que les auteurs postmodernes recourent si souvent à une écriture autobiographique ou autofictionnelle. Peu importe si La canicule des pauvres contient des éléments autobiographiques ou pas. Beaucoup plus intéressant nous semble la manière dont Jean-Simon DesRochers thématise le narcissisme et l'obsession de soi-même. Monique, la propriétaire (sic) du Galant est l'incarnation même du narcissisme. Elle est le prototype de la beauté artificielle, car elle a subi une série d’opérations plastiques. Tel Narcisse, elle passe tout son temps à se regarder. Un autre personnage extrêmement égocentrique est Takao qui ne distingue pas vraiment sa vie et son œuvre : il s'adonne à la création d'une bande dessinée autobiographique. Semblablement, Kaviak n’enregistre que lui-même, ses prouesses sexuelles et spirituelles. Tous les trois ont besoin de spectateurs : Monique pour admirer son corps, Takao pour vivre sa vie, Kaviak pour assouvir ses fantasmes. Ces trois personnages clé ne sont pas capables de vivre sans partager leur intimité. En outre, le narcissisme marque le narrateur dont le souci de montrer aux lecteurs sa supériorité sur les personnages est en fait une obsession. Semblablement l'auteur, lui non plus, néchappe pas au narcissisme, puisqu'il suppose un double lecteur modèle. Le premier est un lecteur naif, un consommateur de masse, celui qui, assez paradoxalement, ne lit pas. Le second, par contre, est un lecteur avisé. C’est lui qui doit déchiffrer les jeux intertextuels et hypertextuels de l'auteur, c'est lui qui doit l'admirer pour son intelligence, son instruction, son art scriptural. Si l'auteur ne lui dédie pas son livre, il lui adresse la dédicace : «Et parce que l'ironie dépasse les prétentions à la sagesse, je dédie ce livre à ceux qui ne lisent pas ». L’exhibitionnisme narcissique de l'auteur se manifeste précisément dans de nombreux clins d’oil qu'il fait au lecteur avisé et dans son désir de la reconnaissance de sa supériorité intellectuelle. Pareillement à ses personnages, lui aussi a besoin des spectateurs qui puissent admirer son œuvre. 


\section{Un métarécit}

La liste des pratiques postmodernes employées dans La canicule des pauvres paraît être complète. Les inventaires ainsi que le zapping audiovisuel, auditif et visuel reflètent le fait que la société actuelle est capable de consommer absolument tout, même les expériences et les souvenirs, et cela sans un moindre souci hiérarchique. Faisant référence à un sac de chips, l'un des symboles de consommation, la composition de l'œuvre et l'écriture fragmentale rendent également compte de l'état de la société qui vit sans mode d'emploi et où le chaos et l'aléatoire l'emportent sur le système ou la symétrie. L'idée du chaos postmoderne est d'ailleurs également liée au métissage : les genres et les langues défilent sans aucune hiérarchisation. Les commentaires du narrateur narcissique et cynique, la représentation des personnages des créateurs pareillement narcissiques et la mise en abyme du projet du livre mettent à nu l'autoconscience du texte qui témoigne d'une société obsédée par elle-même. Par les allusions à la culture de masse, l'intertextualité renvoie à la société de consommation; par les jeux intertextuels et hypertextuels elle contribue à la révélation de son narcissisme et de son autoconscience. Par le choix conscient des hypotextes, La canicule des pauvres se propose comme un successeur des romans-modèles ainsi que l'incarnation du roman postmoderne. La nouvelle lisibilité est atteinte grâce au retour à l'histoire qui entraîne la revisitation de nombreux genres dont le choix évite toute l'hiérarchisation ; grâce au retour au sujet narcissique représenté par les personnages, le narrateur et même l'auteur; et grâce au retour à la réalité qui se révèle crépusculaire, faisant preuve d'un dysfonctionnement et d'une crise profonde. Nous ne pouvons que confirmer l'affirmation de la quatrième de couverture : "Dans cet imposant premier roman, Jean-Simon DesRochers réussit un tour de force : donner à lire une réalité aussi crue que drôle, un monde tellement vivant que sa décadence ne cesse de nous séduire ".

La présence surabondante des pratiques postmodernes dans La canicule des pauvres nous amène à la conclusion que Jean-Simon DesRochers a voulu fabriquer un parfait roman postmoderne, osons dire un métaroman postmoderne. Bien qu'il n'apporte aucune réflexion explicite sur la littérature postmoderne, il devient cette réflexion en se proposant en tant que roman-modèle. Tout en rendant compte d'une ville postmoderne dont Montréal est un excellent représentant, le roman rend compte surtout de la littérature postmoderne dont il se veut un exemple à suivre, lu par la masse qui ne lit pas, étudié par la critique littéraire et copié par la postériorité.

\section{Références bibliographiques}

Dällenbach, L. (1977). Le récit spéculaire. Essai sur la mise en abyme. Paris : Seuil.

Desrochers, J.-S. (2009). La canicule des pauvres. Montréal : Les herbes rouges.

Eco, U. (1985). Apostille au "Nom de la rose». Paris : Grasset.

Genette, G. (1982). Palimpsestes (La littérature au second degré). Paris : Editions du Seuil. 
Gontard, M. (2003). Le roman français postmoderne. Publié le 16 mars 2005. Disponible en ligne : https:// halshs.archives-ouvertes.fr/halshs-00003870 (consulté le 20 avril 2015).

Magné, B. (1998). Perecollages 1981-1988. Toulouse : Presses Universitaires du Mirail.

Paterson, J. M. (1982). L'autoreprésentation : formes et discours. Texte, 1, 177-194. 